\title{
Welcome, ICBS!
}

\section{The launch of the International Chemical Biology Society represents an important step for chemical biology.}

On the fifth anniversary of Nature Chemical Biology, we noted that 'chemical biologists need to create and implement plans that will enable chemical biology to transition from an interdisciplinary research field into an independent scientific discipline' (Nat. Chem. Biol. 6, 385, 2010). Thus, we are pleased to see the creation of the International Chemical Biology Society (ICBS, http://chemical-biology.org/).

The first official ICBS conference (http://www.chemical-biology.org/ conference2012), which was held recently in Cambridge, Massachusetts, brought together an international group of chemical biologists for not only scientific exchange but also in-depth discussions about the objectives of the ICBS and the infrastructure necessary to achieve them. In the past two years, the ICBS leadership has set up a good organizational framework for the society. Yet, guaranteeing the long term success of ICBS will require the enthusiastic engagement of chemical biologists from across the globe, and this depends primarily on the society's ability to be inclusive of the entire discipline and to articulate a vision for how the organization can best serve the field.

To ensure that all chemical biologists find a professional home in the society, it is critical that ICBS embraces the broadest possible definition of chemical biology. Nature Chemical Biology's functional definition-chemical biology includes all research at the interface of chemistry and biology-may serve as a useful starting point. Research that focuses on small molecules, chemical probes and drug discovery remains a defining element of chemical biology and has been well represented by the ICBS thus far. Yet, the diversity of basic and applied research at the chemistry-biology interface has expanded in recent years (Nat. Chem. Biol. 6, 847-856, 2010), which means that a broader view of chemical biology must be integrated into the mission and strategy of ICBS to ensure full participation by the community.

The ICBS must also take an inclusionary approach to chemical biology researchers themselves, ensuring that the society's leadership and programs reflect the communities they serve. As a twenty-first- century organization, the ICBS has a unique opportunity to forge a modern scientific society that represents and unites its members across geographical, racial or gender divides that may have not been sufficiently bridged in professional societies in earlier eras.

Finally, the society needs to articulate a long-term vision for how it will support its members and advance chemical biology on a global scale. The traditional services of a professional society such as conferences, educational initiatives, public outreach and professional development that are currently being considered will provide important value to society members and the general public. However, to maintain a focus on the needs of chemical biology as a discipline, ICBS needs to gather 'grassroots' ideas from the community to shape the direction of the society and identify how it can help chemical biologists translate their goals into action.

We commend the ICBS leadership on their efforts thus far and look forward to seeing how the society develops between now and the next meeting of the ICBS being held in Kyoto, Japan in the fall of 2013.

\section{Introducing Online Methods}

\section{The Methods section of original research papers will be published online only starting in 2013.}

Online publishing platforms and mobile devices have accelerated the migration of scientists from printed journals toward digital formats. As this trend continues, scientific papers will transform into fully integrated and interactive digital entities. Nature Chemical Biology is taking a step toward this future by publishing 'Online Methods' for all of our original research content.

Until now, the methodological detail in Nature Chemical Biology papers has been divided between two sections: Methods that were included in the print and online versions of papers and Supplementary Methods that were published online only. This separation required additional work for authors and made it more difficult for readers to find relevant methodological information. Beginning with our January 2013 issue, Brief Communications and Articles will be published with an Online Methods section that contains the primary methods of the paper in one place.

The adoption of Online Methods, which has been used by other Nature journals in recent years, has several advantages. First, unlike Supplementary Methods, Online Methods will be copy edited by our inhouse team, which facilitates a consistent presentation across our papers. Second, Online Methods save space, giving us more room to publish chemical biology research and commentary in each issue. Finally, the new format better integrates methods across the HTML and PDF versions of the paper. Readers who continue to enjoy the journal in print will be able to access the methods in one of these digital formats at the Nature Chemical Biology website.

The move to Online Methods will affect the formatting of Articles and Brief Communications and their associated Supplementary Information. Authors should continue to follow our established limits to references cited in the body of the paper, but each paper will be allowed additional citations that are associated exclusively with Online Methods. Authors who wish to present extensive descriptions of experimental procedures that may be less suited to Online Methods-complex protocols or detailed information on chemical compound synthesis and characterization-may organize these into a 'Supplementary Note'.

The transition to Online Methods should be seamless for our authors and readers. Authors who plan to submit new manuscripts should review our formatting guidelines in our Guide to Authors (http:// www.nature.com/nchembio/authors/ index.html). As always, our editors will work closely with authors to optimize the presentation of their revised manuscripts for timely publication. We hope that our updated approach to methods will enhance your experience of our published papers. 